\title{
Editorial: Collaborative Computing for Data-Driven Systems
}

\author{
Xinheng Wang ${ }^{1} \cdot$ Muddesar Iqbal $^{2} \cdot$ Honghao Gao $^{3} \cdot$ Kaizhu Huang $^{4} \cdot$ Andrei Tchernykh $^{5}$
}

Published online: 26 December 2019

(C) Springer Science+Business Media, LLC, part of Springer Nature 2019

\section{Editorial:}

Over the last few years, owing to the development, deployment, and use of Internet of Things (IoT) systems and smart devices, a large volume of data has been generated from various operation systems. High speed 4G networks and low cost of data usage foster the commercialization of a few heavy data driven social networks, such as Tik Tok and Instagram. With the commercialization of $5 \mathrm{G}$ networks and guarantee of transmission of large volume of data with short delay, more applications could be developed over the next few years to change the way we live and work. This fundamental change needs to be considered by academics and industry experts for designing and developing new system architecture, new data processing methods, and also new ways of providing quality services to end users to improve the quality of life. This special issue addresses the challenges of this new trend. It features six selected high-quality papers, ranging from new methodology to handle the data and make better decisions, better data processing methodologies to process the data inside the system and across the systems, methods to process the low-quality but complex data generated from the end users, which is normally the case for crowdsensing systems, to cost-effective ways to provide services to benefit both service providers and end users.

In large-scale data-driven systems, how to make a rapid decision is a complex step. Thompson Sampling is one of the effective approaches to balance the explorationexploitation trade-off and make good decisions. However, in dynamic environments, the environment will undergo

Xinheng Wang

xinheng.wang@xjtlu.edu.cn

$1 \quad$ Xi'an Jiaotong-Liverpool University, Suzhou 215123, China

2 London South Bank University, 103 Borough Rd, London SE1 0AA, UK

3 Computing Center, Shanghai University, Shanghai, China

4 Xi'an Jiaotong-Liverpool University, Suzhou, China

5 CICESE Research Center, Ensenada, Mexico frequent and unpredictable changes, which makes the Thompson Sampling difficult to work. In the first paper titled "Collaborative Thompson Sampling", a radical collaborative approach is proposed to address the challenges faced in the dynamic environments, where users are dynamically clustered into groups and the feedback from users in the same group is used to facilitate an optimal choice. This approach can significantly accelerate the convergence of the algorithm and improve the prediction performance.

The second selected paper is "Double-Arc Parallel Coordinates and its Axes Re-ordering Methods". It addresses two challenges of high-dimensional data inside one system, visual clutter and data clarity, by proposing a new visualization method based on Arc Coordinates Plot (ACP). Optimization is also applied to improve the performance in visualizing multivariate dataset and visual experience.

The third paper, "A Two-Stage Approach for Social Identity Linkage based on an Enhanced Weighted Graph Model", however, addresses the challenges of utilizing the heterogenous systems. Over the last few years, the number and size of social networks are growing continuously across the world. A large volume of data is generated from these networks. How to track a person across different networks, i.e., to track a terrorist, is becoming a very difficult task. In this paper, a two-stage approach is proposed to identify the linkage and follow it to other networks by a new method to represent the missing attributes after crossing the networks. This makes the tracking from impossible to possible.

With continuous development and deployment of IoT systems, data is always generated from end users. The quality and complexity of the data make the effective and efficient usage of the data difficult. How to extract knowledge from the lowquality data is a challenge to all the applications. The paper "Adaptive Extraction and Refinement of Marine Lanes from Crowdsourced Trajectory Data" demonstrates the approaches and effectiveness of the approaches by processing crowdsensed data in marine applications. It is an example from low-quality to high-quality by effective data processing. On the other hand, rather than considering the quality of the data as in "Adaptive Extraction and Refinement of Marine Lanes from Crowdsourced Trajectory Data", the complexity 
of the data is considered in the paper titled "Traffic Volume Prediction based on Multi-sources GPS Trajectory Data by Temporal Convolutional Network", where the nonlinearity of the data and contextual data are considered and an artificial intelligence algorithm is applied for predicting the volume of traffic.

Cloud computing is becoming a de facto platform and approach to store and process data, and provide services. However, how to utilize the resources and provide better services is still a challenge. The last paper titled "Cloud Marginal Resource Allocation: A Decision Support Model" addresses this challenge by proposing a marginal resource allocation method to meet the needs of consumers. The proposed method is particularly useful to small- and medium-sized service providers to optimize the use of resources and increase the profits but to reduce the cost of consumers.

Acknowledgements The guest editors are thankful to our reviewers for their efforts in reviewing the manuscripts. We would like to sincerely thank the Editor-in-Chief, Dr. Imrich Chlamtac, for his supportive guidance during the entire process. We would also like to thank the office staffs, Leonora M. Panday and Jed Joseph Adel, for their continuous support in editing this special issue.

Publisher's Note Springer Nature remains neutral with regard to jurisdictional claims in published maps and institutional affiliations.

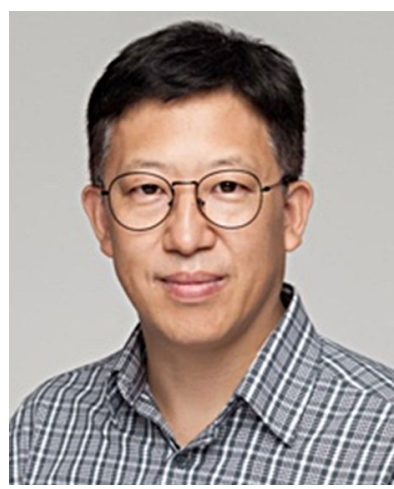

Xinheng Wang (M'04 -SM'14) received the B.E. and M.Sc. degrees in electrical engineering from Xian Jiaotong University, Xian, China, in 1991 and 1994, respectively, and the $\mathrm{Ph} . \mathrm{D}$. degree in electronics and computer engineering from Brunel University London, Uxbridge, U.K., in 2001. He is currently a Professor with the Department of Electrical and Electronic Engineering, $\mathrm{Xi}$ 'an Jiaotong-Liverpool University, Suzhou 215123, China, and an adjunct professor of Zhejiang University. He has been an Investigator or Co-Investigator of more than 20 research projects sponsored from EU, UK EPSRC, Innovate UK, China NSFC, and industry. He has authored or coauthored over 150 referred papers and held 15 granted patents, including 1 US, 1 Japan, 4 South Korea and 9 China patents. His current research interests include Tactile Internet, indoor positioning, Internet of Things (IoT), and big data analytics for intelligent services, where he has developed the world's first smart trolley with Chigoo Interactive (www.chigoo.net). His research has led to a few commercial products in condition monitoring, wireless mesh networks, and acoustic localization and user-centric routing and navigation (www.ciptechnology.co.uk).

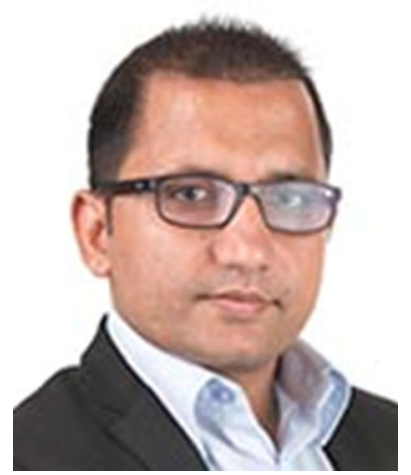

Muddesar Iqbal Dr Iqbal won an EPSRC Doctoral Training Award in 2007 and completed his PhD from Kingston University in 2010 in Wireless Networks. He has been a principal investigator, co-investigator, project manager, coordinator and focal person of more than 15 internationally teamed research and development, capacity building and training projects. In September 2008, he acquired the Status of Foreign Expert from the State Administration of Foreign Experts Affairs, People's Republic of China. He is member of British Computer Society and also a British Council's Active Citizen for the implementation of British Council Social Action Projects under Youth, Education and Society. He is serving as a Member Board of Governor for United Nation's Independent Monitoring Association for Child Labour (IMAC), which was set up by the International Labour Organisation's International Programme on the Elimination of Child Labour (IPEC) from the Sailkot's soccer ball industry in Pakistan.

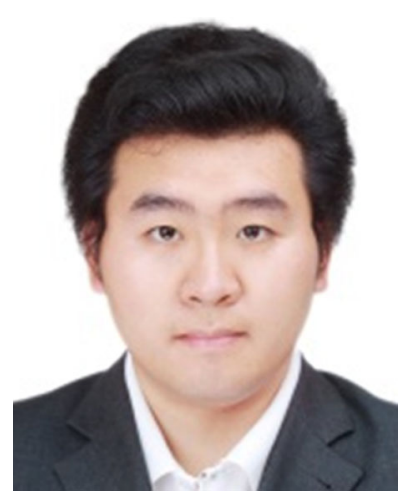

Honghao Gao Prof. Gao received the $\mathrm{Ph} . \mathrm{D}$. degree in Computer Science and started his academic career at Shanghai University in 2012. He is an IET Fellow, BCS Fellow, EAI Fellow, IEEE Senior Member, CCF Senior Member, and CAAI Senior Member. Prof. Gao is currently a Distinguished Professor with the Key Laboratory of Complex Systems Modeling and Simulation, Ministry of Education, China. His research interests include service computing, model checking-based software verification, and sensors data application.

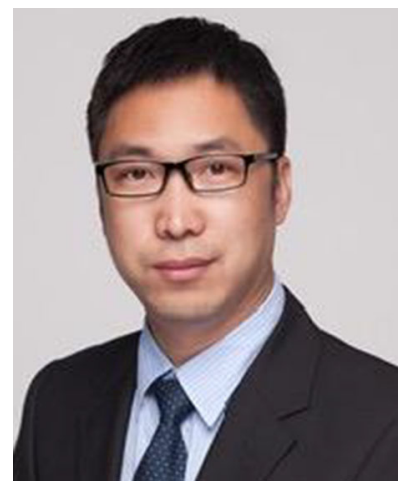

Kaizhu Huang is currently a Professor at Xi'an JiaotongLiverpool University, China. He is also the founding director of Suzhou Municipal Key Laboratory of Cognitive Computation and Applied Technology. Prof. Huang has been working in machine learning, neural information processing, and pattern recognition. $\mathrm{He}$ was the recipient of 2011 Asia Pacific Neural Network Society (APNNS) Younger Researcher Award. He received best paper or book award five times. Until October 2019, he has published 8 books and over 160 international research papers $(60+$ international journals $)$ e.g., in 
journals (JMLR, Neural Computation, IEEE T-PAMI, IEEE T-NNLS, IEEE T-BME, IEEE T-Cybernetics) and conferences (NeurIPS, IJCAI, SIGIR, UAI, CIKM, ICDM, ICML, ECML, CVPR). He serves as associated editors in four international journals and board member in three international book series. He has been sitting in the grant evaluation panels in Hong Kong RGC, Singapore AI programmes, and NSFC, China. He served as chairs in many international conferences and workshops such as BICS, ICONIP, AAAI, ACML, ICDAR, ACPR, and SDA.

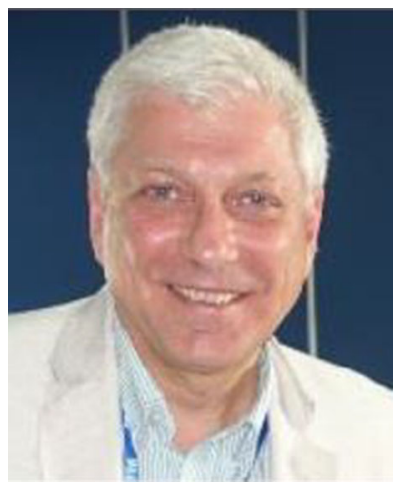

Andrei Tchernykh is holding a full professor position at CICESE Research Center, Mexico and Adjunct professor at Institute for System Programming, Russia. He is chairing "Parallel Computing Laboratory" at CICESE and "Laboratory of Problem-Oriented Cloud Computing" at South Ural State University, Russia. He also is the founding member of the Mexican Supercomputer Society (RedMexSu) and member of the Mexican National Researchers System SNI level II. He has been a principal investigator and coordinator of research projects and grants in different countries funded by CONACYT, NSF, ANII, Ochoa, INRIA, FNR, UC MEXUS, DAAD, LAFMI, AMEXCID, ANII, etc. He has published over 200 papers in the most distinguished scientific journals and international conferences and served as a TPC member, and general co-chair of more than 250 professional peer-reviewed conferences. He has graduated $38 \mathrm{Ph} . \mathrm{D}$. and M.S. students, and served as the External Examiner for Ph.D. and Master programs in India, Malaysia, Germany, Luxembourg. Uruguay, Colombia, México, and France. He has awarded Global Scholars Fellow at Tsinghua University, German Academic Exchange Service fellowship at University of Göttingen, Dortmund University, and Severo Ochoa fellowship at Barcelona Supercomputing Center. He was an Invited Visiting Researcher at INRIA-Lille, UGA, UNI.LU, Grenoble INP, UC-Irvine, USC, UJF, UdelaR. He served or is serving as an Editor or/and Guest Editor for journals, such as the IJMHeur, SUPERFRI, SUSU Vestnik CMI, International Journal of Approximate Reasoning, Programming and Computer Software, MONET. He is engaged in extensive research on parallel, distributed, and cloud computing research addressing multi-objective resource optimization, both, theoretical and experimental, security, uncertainty, scheduling, heuristics and meta-heuristics, adaptive resource provisioning, energy-aware algorithms, and Internet of Things (http://usuario.cicese.mx/ chernykh/). 